\title{
La società italiana e il servizio sanitario nella Pandemia: temi e ricerche
}

\author{
Micol Bronzini*, Stefano Neri**
}

\section{Introduzione}

Prima del diffondersi della Pandemia di Covid-19, in pochi tra i sociologi della salute e della medicina si erano occupati della riemersione di malattie virali e di epidemie". A questa "miopia" collettiva, fa da contraltare il proliferare di studi, volumi collettanei e special issues usciti in questi mesi (Corposanto, Fotino, 2020; Favretto, Maturo, Tomelleri, 2021; Vicarelli, Giarelli, 2021), nel nostro come negli altri paesi (Lupton, Willis, 2021). Contributi che testimoniano un comune senso di impellenza, di necessità, seppure non scevro da una altrettanto condivisa sensazione di disagio nel tentare di cogliere nel suo stesso divenire un fenomeno così complesso, rispetto al quale si è al contempo osservatori e protagonisti. Rivendicano altresì il ruolo pubblico della sociologia in un momento in cui la scena sembra essere il campo esclusivo di microbiologi, virologi, infettivologi ed epidemiologi. Da un lato, infatti, vi è l'esigenza di ripensare le organizzazioni sociali affinché siano più preparate a fronteggiare situazioni analoghe. Dall'altro, occorre favorire processi di resilienza generativa e trasformativa che affrontino quei fattori più distali alla base della vulnerabilità dei nostri sistemi sociali, anche approfittando delle risorse resesi disponibili con il Piano Nazionale di Ripresa e Resilienza (Pnrr).

Questo numero monografico di Salute e Società si aggiunge ai lavori che l'hanno preceduto nel voler contribuire a una lettura assieme "multilivello" e "multifocale" (Favretto, Maturo, Tomelleri, 2021), approfondendo la comprensione di alcuni risvolti dell'emergenza sanitaria e sociale. Il volume raccoglie le riflessioni sviluppate nell'ambito del Convegno della sezione di Sociologia della Salute e della Medicina dell'Associazione Italiana di Sociologia dal titolo «Vivere nell'emergenza: la società italiana e il Servizio Sanitario Nazionale di fronte alla pandemia da Covid 19», che si è tenuto online il 14-15 settembre 2020. Replicando la struttura del convegno, il

*Università Politecnica delle Marche.m.bronzini@univpm.it

*** Università degli Studi di Milano. stefano.neri@unimi.it

${ }^{1}$ Una eccezione, che molti hanno (ri)scoperto in questi mesi, è il numero monografico di Sociology of health and illness del 2013, 35.

Salute e Società, XX, 3/2021, ISSN 1723-9427, ISSNe 1972-4845

DOI: 10.3280/SES2021-002-S1001 
numero è articolato di fatto in due parti. La prima indaga a livello microsociale e macro-culturale l'impatto della pandemia sulla vita quotidiana, con particolare riguardo alle ricadute in termini di salute e benessere. La seconda analizza a livello macro-politico/istituzionale e meso organizzativo/professionale le caratteristiche strutturali con cui il sistema sanitario italiano si è trovato a gestire l'emergenza sanitaria, nonché, di riflesso, l'impatto di quest'ultima sui suoi elementi costitutivi.

Oltre a portare l'attenzione su dimensioni e temi differenti, i diversi saggi mostrano la ricchezza di approcci teorici che la nostra disciplina può offrire all'interpretazione dei processi in atto: dalle tesi sulla società del rischio alle teorie sulla costruzione dei problemi sociali attraverso la comunicazione pubblica, dall'analisi foucaultiana dei processi di governamentalità al realismo critico, dagli approcci critici verso la sanità digitale alla political economy, per citarne alcuni. Mai come oggi vi è la necessità di una sociologia della salute e della medicina critica, capace di de-costruire i discorsi dominanti, di dare voce alle istanze di coloro che sono meno visibili e di fornire indicazioni di policy "coraggiose", che contribuiscano a ripensare individui, famiglie, comunità e sistemi di salute.

\section{Fratture e rammendi nei vissuti della pandemia}

Il Rapporto annuale Istat 2020 sulla situazione del Paese mostra una fotografia della società italiana durante il lockdown abbastanza confortante, descrivendola come fortemente coesa, con livelli di fiducia istituzionale (soprattutto verso i professionisti sanitari) altissima, elevato senso civico, prevalenza di un clima sereno e positivo nell'ambito famigliare, vissuto come rifugio. Lo stesso documento segnala una serie di elementi favorevoli associati al lungo confinamento domestico: la maggiore condivisione delle responsabilità di cura tra partner, la tenuta delle relazioni sociali ancorché a distanza, il mantenimento dell'attività fisica, la riscoperta della lettura e degli hobbies. Già dal Rapporto emergono, però, anche possibili criticità, a partire dal rischio che la convivenza forzata porti a un incremento della violenza di genere. Si sottolinea altresì la polarizzazione dei comportamenti sociali: da un lato, coloro che hanno potuto dedicarsi maggiormente a sé stessi e al proprio progetto di vita, valorizzando le opportunità di riflessione intima, di ricarica personale, di crescita, di cambiamento; dall'altro, quanti hanno esperito un intensificarsi delle relazioni sociali e di cura; infine, quanti hanno sofferto vissuti di solitudine, vuoto, chiusura, stallo, stress, ansia. Sul fronte degli esiti di salute, i primi dati del Rapporto confermano un acuirsi delle 
diseguaglianze sociali che contraddistinguono il nostro Paese, con un eccesso di mortalità da Covid-19 tra le persone in condizione di svantaggio socio-economico.

A distanza di alcuni mesi, complice la cronicizzazione della situazione emergenziale, gli elementi che destano preoccupazione sembrano accresciuti. Le ricerche sull'impatto della pandemia nella sfera psicologica ed emozionale rilevano un aumento generalizzato di ansia, stress e disturbi depressivi, fronteggiato con una maggiore assunzione di ansiolitici, sonniferi e antidepressivi (Camilla, 2020; Caritas, 2020; Rita, 2021). Alcuni sostengono si configuri una vera e propria nuova condizione clinica: lo stress da pandemia (Biondi, Iannitelli, 2020). A ciò si aggiunge la cosiddetta Covid fatigue, ossia un incremento della fatica percepita nella sfera lavorativa per gli adulti e un calo della concentrazione nello studio per ragazzi e giovani.

Sul fronte famigliare, i dati Istat (2021) e quelli degli uffici giudiziari attestano, rispettivamente, un significativo aumento delle chiamate al numero antiviolenza (aumentate del 79,5\% nel 2020 rispetto al 2019 con 1' $8,6 \%$ delle donne che ne attribuisce la causa a situazioni famigliari legate alla pandemia) e dei procedimenti iscritti per reato di maltrattamento. Inoltre, secondo le associazioni di settore, sarebbero in forte crescita le richieste di separazioni e divorzi dovute alla convivenza forzata, con stime che oscillano tra il $+30 \%$ e il $+60 \%$ (sebbene non confermate, almeno sinora, dai dati Istat).

Sul piano comunitario, a minare la coesione sociale, ben al di là delle spaccature riportate nelle cronache quotidiane (in primis quella sul vaccino diventato elemento di forte conflittualità), è l'incremento di nuovi poveri e l'acuirsi delle diseguaglianze sociali (Caritas, 2020; Oxfam, 2021). Contraddicendo la presunta "democraticità" del virus, la pandemia, sia nelle sue conseguenze sanitarie che sociali, ha colpito e continuerà a colpire di più coloro che hanno minori risorse biologiche, psicologiche, sociali, culturali, spaziali e materiali. Tali gruppi sociali sono maggiormente esposti non solo al rischio di contrarre la malattia in forme più gravi ma anche, e soprattutto, alle conseguenze di medio-lungo periodo delle misure di contenimento (che, come noto, penalizzano soprattutto gli autonomi, i lavoratori precari, saltuari e irregolari) e della crisi economica; tutto ciò ha portato a ri-soprannominare il Sars-CoV2 come il "virus della disuguaglianza".

I dati sinteticamente richiamati colgono alcune delle manifestazioni più acute dell'impatto della pandemia sul tessuto sociale; rimane, però, in ombra il ben più pervasivo effetto sulla salute individuale, famigliare e comunitaria, legato non solo alle sue conseguenze concrete, ma anche alla messa in discussione di precedenti sistemi di senso e di significato. In un bel saggio del 2011 Caroline Hovanec, a partire da tre diverse opere letterarie degli anni 
'30 sulla Spagnola, offre alcuni spunti interpretativi sulle fratture, materiali e simboliche, prodotte dal diffondersi del virus su tre diverse unità sociali l'individuo, la famiglia e la comunità - e sulle conseguenti esigenze di riparazione. Ci sembra utile ripartire da alcuni aspetti evidenziati nella sua analisi, sebbene con la consapevolezza di differenze significative di contesto.

A livello individuale, il virus minaccia il senso di integrità dell'individuo, la presunta invalicabilità di quelle difese erette in nome della sacralità del corpo, la percezione di controllo sulle sue aperture di comunicazione e scambio. Chi è stato infettato spesso racconta l'esperienza spaesante di un corpo percepito improvvisamente come "alieno" - una percezione che peraltro può rimanere anche una volta guariti a causa della difficoltà a recuperare l'olfatto e del persistere di uno stato generale di stanchezza e affaticamento, fisico e mentale; se il vissuto di estraneità del proprio corpo è comune a molte altre esperienze di malattia, quello che contraddistingue la Covid-19 è l'immaginario simbolico di ibridazione tra umano e non umano, accentuato dal trattarsi di un virus animale che ha compiuto il cosiddetto "salto di specie"2. L'insicurezza rispetto al proprio corpo e alla sua possibile violazione da parte di un pericolo invisibile e diffuso finisce con il pervadere anche chi non ha contratto il virus, ma quotidianamente sperimenta la condizione liminale di malato potenziale. Il tentativo di difesa da questo senso di minaccia, di disintegrazione personale si esprime in una estrema medicalizzazione della vita quotidiana, a livello concettuale, interazionale e organizzativo-istituzionale (Conrad, 2007).

Sul fronte famigliare, la disgregazione (concreta e/o simbolica) si ricollega all'aver riconcettualizzato anche le relazionali famigliari come possibile vettore di contagio. Quando «la famiglia diviene non un riparo dal pericolo, ma un pericolo essa stessa» (Hovanec, 2011, p. 174) anche le normali espressioni di affettività e cura, come il contatto fisico, generalmente indice di armonia e unità, vengono minate. Si tratta, dunque, di capire se (ed eventualmente come) siano cambiate le modalità del prendersi cura in/della famiglia. Altro interrogativo è come si ricomponga il senso di integrità che è stato attraversato dalla malattia $\mathrm{o}$, ancor più, da lutti ad essa riconducibili.

Sul fronte comunitario, la pandemia accentua alcune linee di frattura già presenti (geografiche, socio-economiche, culturali) e crea nuove faglie: in questi mesi abbiamo visto emergere molte crepe in quella coesione sociale

${ }^{2}$ Anche i nuovi vaccini a diffusione virale, che utilizzano come vettore una versione modificata dell'adenovirus degli scimpanzé, rinforzano questa idea di corpi ibridi (un elemento simbolico che può contribuire allo scetticismo e alle preoccupazioni verso questa nuova tecnologia). 
rilevata dall'Istat all'indomani del lockdown ${ }^{3}$. Da questo punto di vista, la sociologia dei disastri (Mela, Mugnano, Olori, 2017) ci offre utili strumenti di comprensione dell'evolversi delle dinamiche comunitarie di fronte a "stress test" simili, di come la coesione che si registra abitualmente nell'immediatezza dell'evento, possa lasciare campo, con il procedere del tempo, a spaccature, conflitti, rivendicazioni, colpevolizzazioni. Come si rammenda il tessuto sociale e si ricompongono le comunità? Al riguardo, possono venirci in aiuto le molte ricerche sul Terzo settore e sulle nuove forme di solidarietà che si sono intessute in questo anno e mezzo e che hanno permesso la tenuta comunitaria.

Tra le tante spaccature che si sono aperte con il passare dei mesi, una ci sembra particolarmente preoccupante: quella che investe il legame tra generazioni. Nel giro di pochi giorni, si è passati dalla retorica dell'invecchiamento attivo a quella della vulnerabilità degli anziani: le stesse persone che fino a poco prima erano sollecitate a mantenersi in forma, coltivare relazioni sociali e, perché no, restare produttive, si sono riscoperte fragili, da tutelare al caro prezzo della rinuncia alla socialità e alle attività al di fuori delle mura domestiche. Come recuperare una concezione positiva della vecchiaia, dopo la svalutazione che si è registrata a più riprese nel discorso pubblico? Come affrontare le nuove tensioni intergenerazionali legate alle limitazioni richieste agli uni a salvaguardia degli altri? Se è vero, come scrive Luigi Pellizzoni (2020, p. 7), che la risposta a pestilenze e catastrofi è sempre stata la ricerca di «forme ritualizzate e sistemi di senso capaci di mantenere il legame sociale nelle condizioni più avverse, evitando che il forte prevalesse sul debole e che il sacrificio di qualcuno per il bene di altri si compisse nella solitudine dell'anima», ci sembra che oggi ci sia scarsa attenzione a questi aspetti.

Le fratture prodotte (e/o amplificate) a livello individuale, famigliare e comunitario richiedono un attento lavoro di "rammendo e di riparazione" (Cozza et al., 2020). Bisogna peraltro considerare che la pandemia ha investito una società già profondamente fratturata (Scambler, 2020) e disgregata, a tutti e tre i livelli, per effetto di decenni di immaginario simbolico-culturale e di politiche neoliberiste. Non è un caso, che i tanti studi ormai disponibili fatichino a ricostruire un quadro d'insieme. Un ulteriore elemento su cui le analisi dovrebbero aiutarci a far luce è se davvero siamo in presenza di una rottura dell'organizzazione sociale precedente, in grado di

${ }^{3}$ Il bisogno di comunità ha inizialmente favorito la stigmatizzazione dei pochi (in quel momento) "devianti" (ridefiniti covidiots), con ciò disconoscendo peraltro i determinanti sociali di comportamenti percepiti come più o meno responsabili. 
favorire l'auspicata resilienza trasformativa, o se non assistiamo piuttosto a una accelerazione di tendenze in atto già da tempo, anche sul fronte della vita quotidiana ${ }^{4}$.

\section{Il Ssn nella pandemia tra disgregazione e resilienza}

La pandemia ha posto il Servizio Sanitario Nazionale (Ssn) di fronte ad una sfida di entità fino a quel momento sconosciuta, facendo venire al pettine una serie di nodi che si erano accumulati nel corso degli ultimi decenni (Neri, 2020). Nel corso del 2020, il Ssn ha subito forti spinte alla destrutturazione e disgregazione nella sua natura di sistema. Innanzitutto, la risposta alla prima, rapida diffusione del Covid-19 ha messo in luce una tendenza alla disgregazione funzionale del sistema: i servizi di igiene e sanità pubblica, la medicina generale e l'assistenza territoriale, l'assistenza ospedaliera hanno reagito in modo pressoché indipendente all'emergenza. Con le dovute eccezioni locali, è risultata evidente la carenza se non la mancanza di coordinamento tra i diversi tipi o livelli di assistenza.

Questo elemento ha acuito le fragilità delle diverse componenti del Ssn, fortemente indebolite nel corso degli anni dalle politiche di sottofinanziamento e di austerità verso il Ssn. Ne è risultata, com'è noto, l'incapacità dei servizi di sanità pubblica di tracciare e prevenire i contagi, l'impossibilità dei servizi e degli operatori delle cure primarie di curare $\mathrm{i}$ pazienti a casa e di svolgere quindi una funzione di filtro dei casi più gravi, il sovraffollamento degli ospedali da parte di pazienti spesso giunti al ricovero in condizioni ormai non recuperabili. L'assistenza fornita dal Ssn si è scoperta ancora centrata sui servizi ospedalieri, mentre sono emersi chiaramente tutti i limiti dell'assistenza territoriale e dell'integrazione tra ospedale e territorio, con pesanti conseguenze per i pazienti e la cittadinanza, nonché per la stessa efficacia dei servizi ospedalieri.

Il timore del collasso del sistema sanitario è stato così forte da essere addotto come una delle ragioni principali per il ricorso al lockdown della primavera del 2020 e, poi, per le chiusure e limitazioni della scuola e delle attività economiche dell'autunno inverno 2020-21. Del resto, dopo la revisione dell'estate 2021, anche gli indicatori per l'ingresso delle Regioni

${ }^{4}$ Molte imprese di medio-grandi dimensioni facevano già un crescente ricorso a forme di smartworking, le Università promuovevano forme di didattica a distanza, la mediazione tecnologica dei rapporti sociali era già molto avanzata, così come la privatizzazione del tempo libero (basti pensare alla crisi delle sale cinematografiche dovuta all'esplosione della distribuzione attraverso piattaforme digitali). 
nelle zone (bianca, gialla, arancione e rossa) che definiscono diversi gradi di restrizioni alle attività per contenere la diffusione del contagio misurano prevalentemente la capacità del sistema sanitario e ospedaliero di reggere l'impatto della pandemia, più che la diffusione della malattia in sé.

Alla tendenza alla disaggregazione funzionale si è sovrapposta una spinta alla disaggregazione territoriale del Ssn. La pandemia ha fatto esplodere le contraddizioni accumulate nella governance del sistema negli ultimi quindici anni (Neri, 2020). Da un lato, essa ha reso evidente la necessità di un coordinamento nazionale per il contrasto del contagio, non solo sotto il profilo prettamente sanitario ma anche per le strette connessioni con altri ambiti di policy, dall'ordine pubblico, alle attività produttive, alla scuola 0 ai trasporti. Dall'altro lato, il ruolo delle Regioni nell'organizzazione e nella gestione dell'assistenza sanitario è emerso agli occhi dei cittadini con una chiarezza sconosciuta in passato.

In primo luogo, le Regioni hanno inciso fortemente sulla definizione delle politiche nazionali contro la pandemia, almeno dalla fine del lockdown nel maggio 2020 (Capano, 2020; Vicarelli, Neri, 2021), in un processo di negoziazione continua con lo Stato, tra cooperazione e conflitto. Inoltre, sul proprio territorio le Regioni hanno spesso approvato misure di contrasto alla pandemia in ordine sparso e in modo non coordinato né con lo Stato né tra loro. In una situazione nuova e largamente imprevista, il rischio di deflagrazione del Ssn è stato in alcuni momenti evidente, quale prodotto finale di due esigenze difficilmente componibili, ossia un maggiore coordinamento centrale e una differenziazione territoriale delle politiche di contrasto al contagio.

Infine, soprattutto nella prima ondata della pandemia, sono stati messi in discussione i principi universalistici e solidaristici del Ssn, con il rischio di una disgregazione valoriale del sistema. Oltre alle tradizionali diseguaglianze nell'accesso ai servizi e nella salute di natura territoriale e socio-economica, è emerso il tema del razionamento legato all'anzianità e alla fragilità dei pazienti. Forme di razionamento delle risorse e delle prestazioni sono sempre esistite, ma l'emergenza ha indotto a parlare esplicitamente di una discriminazione per età dei pazienti e, a quanto pare, anche a praticarla in qualche caso, dando priorità alle cure dei più giovani $\mathrm{e}$ meno fragili. Vanno inserite in questo quadro anche la forte sottovalutazione iniziale della gravità della situazione nelle Rsa e, per molti versi, anche la scelta di differire le prestazioni diagnostico-terapeutiche di patologie differenti dal Covid, che riguardano prima di tutto anziani e pazienti affetti da plurimorbilità. 
Fortunatamente, nel corso del 2020 e, soprattutto, nel 2021 il Ssn ha imparato a fronteggiare meglio la pandemia e le spinte alla disgregazione sono state contenute e, almeno per il momento, allontanate. Come attestato anche da autorevoli istituzioni (Corte dei Conti, 2021), il sistema ha tenuto, mostrando una considerevole capacità di resistenza e adattamento alla nuova situazione, o di resilienza, come è stato spesso detto. Seppure con un carattere per lo più emergenziale e poco innovativo (Vicarelli, Neri, 2021), i provvedimenti emanati dai governi nazionali nel 2020-21 e il Piano Nazionale di Ripresa e Resilienza hanno delineato un programma di rafforzamento dei diversi livelli di assistenza, identificando le cure primarie e la medicina di comunità come un ambito prioritario di intervento, in linea con la letteratura internazionale (Oecd, 2021), e sforzandosi di promuovere l'integrazione tra ospedale e territorio. Nella stessa linea, sembrano muoversi alcuni dei sistemi sanitari regionali messi maggiormente sotto accusa dalla pandemia, come quello lombardo; altri, come quello del Lazio, oggetto di massicce restrizioni per effetto dei piani di rientro dal debito, hanno rivelato un'insospettabile capacità di risposta nell'emergenza e nel programma vaccinale.

Le relazioni tra Stato e Regioni hanno trovato un nuovo equilibrio nel corso del 2021, favorito dalle condizioni politiche di "unità nazionale" determinatesi con il governo Draghi entrato in carica nel febbraio 2021 e dalla maturazione di processi di apprendimento alla cooperazione avviati nel 2020 (Baldi, Profeti, 2020). La tendenza ad un regionalismo "autarchico" da parte delle Regioni (Caruso, 2021) ha poi trovato un limite nella sentenza della Corte Costituzionale n. 37 del 24 febbraio 2021, pubblicata il 12 marzo 2021, che ha riaffermato la competenza legislativa esclusiva statale nell'emanazione delle misure di contrasto alla pandemia. Infine, anche sotto il profilo valoriale, i principi universalistici e solidaristici del Ssn sembrano essere stati riaffermati, con una nuova attenzione per l'assistenza agli anziani, alle Rsa e ai servizi per la non autosufficienza.

Restano ovviamente numerosi punti interrogativi sulla capacità del processo in atto di invertire in modo duraturo le tendenze operanti nei due decenni precedenti. Il rafforzamento della medicina generale, delle cure primarie e dell'integrazione tra assistenza territoriale e ospedaliera è un obiettivo che si è cercato di perseguire più volte nel corso degli anni passati, con strumenti non molto diversi da quelli attuali e con risultati limitati. La cooperazione tra Stato e Regioni sembra dipendere per molti versi da condizioni politiche non facilmente riproducibili nel tempo e assai legate all'emergenza. La solidità valoriale del Ssn appare ancora incerta, anche perché permane spesso una contrapposizione tra economia e salute in larga 
misura distorta e fuorviante. La disponibilità economica dei finanziamenti del programma Next generation Eu non pare sufficiente a produrre i mutamenti desiderati. Molto dipenderà ovviamente dai rapporti di forza e dalle capacità delle coalizioni degli attori e degli interessi in campo di far prevalere le proprie preferenze e soluzioni.

\section{Il volume}

I contributi raccolti in questo numero ci aiutano a farci strada tra $\mathrm{i}$ molteplici interrogativi posti nei due paragrafi precedenti. Prima di introdurre i saggi che affrontano la dimensione della vita quotidiana, occorre una precisazione rispetto all'arco temporale su cui gli stessi insistono. Le ricerche presentate sono state condotte durante o subito dopo il lockdown primaverile (Marzo-Maggio 2020), in una fase contrassegnata da un profondo sovvertimento tanto del mondo della vita quotidiana quanto delle istituzioni sociali. Nel momento in cui scriviamo, nuovi (in parte) temi già occupano la scena (tra tutti, il green pass e il PNRR); siamo però fermamente convinti dell'utilità di una slow sociology, che si soffermi ancora su quanto avvenuto durante quello straordinario "esperimento di rottura" che è stato il lockdown generalizzato, sulle iniziali strategie di assorbimento e adattamento (Manca, Benczur, Giovannini, 2017) messe in atto tanto a livello individuale che collettivo. Simili analisi permettono, infatti, di mettere a fuoco tensioni e nervature scoperte, di cui è fondamentale avere consapevolezza per non cadere nella rassicurante illusione di un determinismo positivo (Caporale, Pirni, 2020), che prospetti facili automatismi nel tradurre le (pur notevoli) opportunità $\mathrm{e}$ aspettative legate ai finanziamenti del Pnrr in un miglioramento generalizzato del sistema sociale.

Il saggio di Antonio Maturo, Veronica Moretti e Marta Gibin si concentra sui vissuti di coppie con status socio-economico elevato, senza figli, per le quali il confinamento nello spazio domestico è stato per lo più «sinonimo di contenimento e in ultima istanza di libertà» (Cozza et al., 2020, p. 194). Molti sono gli spunti di riflessione proposti alla luce delle due "facce" con cui il lockdown si è presentato: nel medesimo tempo perturbante e performante. L'elemento perturbante risiede nel mescolamento di elementi nuovi in ciò che è familiare e di elementi familiari nel nuovo. Possiamo pensare a quanto vi sia di perturbante nell'aver assistito "in diretta", comodamente seduti nel rassicurante divano di casa, ai bollettini quotidiani della protezione civile e, ancor più, alla parossistica processione di carri militari che attraversavano la città di Bergamo con il loro carico di feretri. Di fronte a tale "perturbazione", 
la risposta che emerge dalle interviste raccolte dagli autori non è solo all'insegna dell'adattamento e della sospensione dell'ordine quale mezzo per salvarlo (Pfister, 2020), ma anche di un suo uso strategico. Colpisce il rifiuto simbolico di tale condizione di interruzione e il continuo rimando al dover (re)agire, fare qualcosa, conservare le proprie routine. Ciò dimostra, come evidenziano gli autori, l'interiorizzazione di quella cultura della prestazione (Chicchi, Simone, 2017), che caratterizza la società neoliberistica tanto sul fronte lavorativo, quanto su quello della cura del corpo, rispetto alla quale anche il lockdown è stato vissuto come un "deceleratore funzionale" (Rosa, 2010). Viene da chiedersi se tale ansia performativa non possa leggersi anche come difesa rispetto alla esposizione quotidiana (ancorché virtuale) alla morte, in ciò riflettendo l'altrettanto consolidato processo di rimozione sociale e culturale della stessa che, come in diversi hanno scritto, la pandemia ha paradossalmente accentuato (Sisto, 2018).

Il contributo di Maddalena Cannito, Benedetta Polini e Alice Scavarda si sofferma sulle coppie con figli in età pre-scolare e scolare, di cui si indagano i processi di riorganizzazione famigliare conseguente al lockdown e, in particolare, al sovrapporsi del lavoro da remoto con le esigenze di accudimento dei figli (acuite nel caso della didattica a distanza). Le autrici sono interessate a capire se vi sia stata una riconfigurazione dei ruoli lavorativi e genitoriali e con quali ripercussioni sulla salute mentale. Quanto emerso dalle due ricerche presentate mostra luci e ombre. Da un lato, i conflitti tra ruolo lavorativo e genitoriale risultano aumentati e si sono riverberati sulla gestione di tempi e, soprattutto, spazi, con ricadute negative in termini di maggiore stress. Dall'altro, si sono ridotti i sensi di colpa, soprattutto per le donne, mitigati dalla maggiore presenza fisica a fianco dei figli. In linea con quanto emerso nel saggio precedente, il profondo sconvolgimento della realtà sociale non ha portato a rivedere i modelli prescrittivi di genitorialità intensiva, radicati nella concezione neoliberista che pone al centro la responsabilità dell'individuo (Sità, 2017), rispetto ai quali gli intervistati si sono adoperati più che altro nel trovare strategie di adattamento. Ad ogni modo, si è reso particolarmente evidente lo scaricarsi in prevalenza sulle donne dei costi della conciliazione e si è evidenziata l'esigenza di ripensare le politiche per la genitorialità affinché sostengano concretamente un modello dual earner-dual carer.

L'articolo di Pietro Paolo Guzzo, Cristina Martini e Giovanni Frazzica si iscrive nel filone degli studi sulla comunicazione attraverso i (vecchi e nuovi) media durante la pandemia, che ha avuto notevole impulso in questi mesi. Gli autori si focalizzano sui lanci stampa dell'Ansa durante il lockdown evidenziando «temi e dinamiche sociali emerse/sommerse/costruite» dal 
«circuito politico-tecnico-mediatico». Gli articoli analizzati propongono tre usi metaforici della pandemia, cui corrispondono diversi modi di rappresentare le dinamiche famigliari, a proiezione interna o esterna: quella da "clima bellico post-apocalittico", quella "segregazionista e normativa" e infine quella "reattiva e partecipata". Tali metafore, nel costruire specifiche rappresentazioni delle realtà e dei problemi sociali, contribuiscono a improntare i modelli di azione delle persone. Come ricordano gli autori, la metafora bellica è servita paradossalmente a iscrivere la situazione che si stava vivendo entro un registro familiare, ancorché tragico, riducendo in ciò l'incertezza e al contempo creando coesione (nonché a rendere accettabili misure restrittive del tutto eccezionali). Molti sociologi, e non solo, hanno da subito messo in guardia rispetto ai limiti e ai rischi del ricorso dominante e fuorviante ${ }^{5}$ a tale metafora, altrove definito come "un inceppamento del linguaggio" (Kirchmayr, 2020). La proposta di altre metafore meno deresponsabilizzanti e il superamento di certe antinomie paradossali si fa, dunque, quanto mai urgente.

Il ruolo giocato dalla comunicazione (e dalla sua mancanza) nell'attivazione di strategie di fronteggiamento personali viene affrontato anche nel saggio di Maurizio Esposito e Lucio Meglio che, adottando una prospettiva micro-relazionale, pongono l'attenzione sul contributo della comunicazione sanitaria alla gestione del rischio. Gli autori, sulla base di interviste a persone guarite dalla Covid-19, riflettono sui messaggi che queste hanno ricevuto e sul loro impatto sui vissuti emotivi e sulla percezione del pericolo. Emergono non solo importanti deficit comunicativi ma anche, in molti casi, i limiti della presa in carico da parte dei medici di medicina generale. La pandemia ha evidenziato una nervatura scoperta nella relazione di cura, che già da tempo veniva denunciata da tante ricerche sui pazienti cronici.

Il contributo di Lia Lombardi, Alessandra Sannella e David Donfrancesco si focalizza, invece, sulle persone in stato di vulnerabilità. L'analisi dei bisogni espressi dai gruppi sociali più fragili rileva come la pandemia abbia compromesso la possibilità di soddisfare bisogni primari, innescandone al contempo di nuovi connessi all'esigenza di garantire la continuità scolastica

${ }^{5}$ La stessa rappresentazione mediatica degli "eroi" di questo tempo (medici e infermieri) oltre a essere manchevole (meno visibili sono stati altri lavoratori essenziali: il restante personale delle organizzazioni sanitarie, cassieri, operatori della logistica e dei trasporti, riders, etc.), dissimula la realtà di professionisti impegnati duramente in contesti organizzativi che non sempre hanno saputo assicurare loro una adeguata protezione dai rischi di contagio, anche per il fallimento di alcune logiche manageriali e di modelli organizzativi (si pensi alle "zero scorte"). 
e lavorativa. La risposta dei servizi (pubblici e del terzo settore) conferma quanto emerso da altre indagini, ossia, una capacità di resilienza adattiva e una rafforzata capacità di svolgere un'azione sussidiaria integrata (Oxfam, 2021). Le strategie innovative con cui il Terzo settore è intervenuto in questa fase sono al centro anche della seconda ricerca-azione volta a rilevare criticità e bisogni espressi dai migranti ospiti dei centri di accoglienza (nonché rappresentazioni culturali della salute e della malattia). Da questi si è partiti per sviluppare un'azione informativa anti-Covid partecipata, nell'ottica della promozione della salute di comunità.

L'articolo di Monica Murero e Giuseppe D'Ancona costituisce in un certo senso il trait d'union tra i saggi della prima parte e quelli che seguono, dal momento che affronta, a partire da una ricerca qualitativa di taglio microanalitico, un aspetto del più ampio processo di digitalizzazione dei sistemi di cura, centrale nelle riflessioni sulla resilienza trasformativa dei sistemi di salute sviluppate nella seconda parte del volume. Il passaggio alla cosiddetta ricetta elettronica viene analizzato dagli autori guardando ai suoi riflessi sulla creazione di "micro-reti di cura digitale" in cui è risultata fondamentale, in alcuni casi, l'intermediazione di "interdigital carers". L'emergere di questi ultimi ha permesso non solo di superare gli iniziali ostacoli, dovuti alla carenza di competenze digitali da parte dei principali destinatari dell'intervento, ossia gli anziani cui si raccomandava di restare a casa, ma anche di diffondere proprio tali competenze. Questo lavoro evidenzia come, al di là di sterili contrapposizioni tra posizioni tecno-pessimiste e tecnoottimiste, sia utile e necessario considerare le pratiche effettive e gli usi situati che permettono di cogliere il ruolo che le tecnologie giocano nella costruzione sociale delle relazioni interindividuali di cura (Vicarelli, Bronzini, 2019).

I contributi dedicati al Ssn si pongono a metà tra l'analisi delle cause delle criticità emerse nella pandemia e le proposte di rilancio del sistema sanitario. Tali lavori proseguono e approfondiscono percorsi di ricerca avviati dagli autori, che per alcuni versi avevano trovato una prima espressione in Vicarelli, Giarelli (2021). Prima di tutto, Guido Giarelli sviluppa un'analisi comparata della capacità di risposta alla pandemia da parte dei sistemi sanitari europei mostrando, mediante dati quantitativi, che non solo l'impatto sulle diverse "macro-regioni" in cui sono raggruppati i sistemi sanitari è stato differente, ma che esiste anche una forte variabilità interna ad ogni macroregione. Per spiegare i risultati ottenuti, è utile fare riferimento non solo al concetto di resilienza, ma anche a quello di vulnerabilità. Dopo avere definito, operativizzato e combinato i due concetti, si arriva a quello di sostenibilità di un sistema sanitario, inteso come la sua capacità di continuare 
a svolgere le funzioni di prevenzione, cura e riabilitazione a favore della popolazione di riferimento, in un ambiente soggetto a continuo cambiamento. L'analisi consente di collocare il caso italiano nel contesto internazionale, fornendo un'interpretazione di quanto accaduto nel nostro paese mediante l'utilizzo di un quadro concettuale ispirato alla migliore letteratura sociologica internazionale. È possibile, infatti, non solo avanzare delle spiegazioni sulle ragioni per cui l'Italia emerga come un "casosentinella", caratterizzato da una forte vulnerabilità alla crisi pandemica, ma anche formulare delle ipotesi sulla capacità del Ssn di passare da resilienza adattiva ad una trasformativa, agendo sulle criticità strutturali del sistema.

Nel saggio successivo, Stefano Neri si chiede se e in quale misura il processo di razionalizzazione dei servizi ospedalieri, ispirato al New Public Management e intrapreso dalla fine degli anni Ottanta, abbia contribuito all'evidente difficoltà del sistema ospedaliero italiano a reggere l'urto della pandemia. Analizzando una serie di dati internazionali e nazionali relativi ai posti letto per acuti e complessivi, nonché ai posti letto nei reparti maggiormente interessati dal Covid-19, emerge che nel caso italiano la riduzione delle risorse strutturali sia stata tale da privare il sistema ospedaliero di risorse (il cosiddetto slack organizzativo) che lo avrebbero messo in grado di fronteggiare l'emergenza con minore sofferenza. E tuttavia, le difficoltà del sistema ospedaliero vanno ricondotte anche alla mancata costituzione di una rete di cure primarie e di comunità in grado di prendere il posto dell' assistenza ospedaliera sul territorio

Al riguardo Carmine Clemente, Anna Rosa Favretto, Angela Genova e Davide Servetti ricostruiscono il processo di riforma delle cure primarie in Italia, dal 2000 ad oggi, concentrandosi sull'introduzione delle Case della Salute e sul loro percorso di implementazione, fino alla previsione delle Case della Comunità nel Pnrr approvato alla fine di aprile 2021. L'analisi di tre casi regionali (Piemonte, Marche e Puglia) fa emergere alcune criticità rilevanti nell'esperienza delle Case della Salute, che vanno ricondotte allo stallo della contrattazione collettiva tra Ssn e medici di medicina generale, nonché ad una tendenza ad assorbire e "metabolizzare" le Case della Salute nell'assetto organizzativo preesistente della sanità territoriale, depotenziandone il carattere innovativo. Gli autori sono però ottimisti sul futuro delle Case della Comunità, contando sulla capacità di un evento come la pandemia di innescare un processo di rottura rispetto alla situazione preesistente favorendo così l'implementazione di riforme non incrementali, aiutata anche dalle cospicue disponibilità finanziarie.

L'analisi comparata dei sistemi sanitari regionali è al centro anche del contributo di Valeria Quaglia, Marco Terraneo e Mara Tognetti. Gli autori 
selezionano tre Regioni (Lombardia, Veneto e Campania), che hanno adottato tre diversi modelli regolativi di sistema sanitario, analizzando le differenze esistenti nella risposta alla prima ondata del Covid e l'impatto di tali differenze sulla salute dei cittadini e del personale sanitario. In particolare, l'analisi quantitativa di dati Inail, Protezione Civile e Istat mostra non solo che l'esposizione al rischio di contagio nel personale sanitario sia stata ovunque più alta rispetto alla totalità della popolazione, ma anche che in Lombardia si sia presentato un eccesso di rischio rispetto agli altri casi esaminati; ciò appare riconducibile principalmente all'elevata ospedalizzazione dei malati di Covid in Lombardia, nei mesi iniziali della diffusione del contagio.

Alla "questione lombarda" nella pandemia, come la chiamano gli autori, dedicano infine il loro contributo Marco Arlotti e Michele Marzulli. Almeno nel 2020, infatti, il sistema lombardo è quello che ha mostrato le maggiori criticità, risultando l'epicentro della crisi. Gli autori chiamano in causa le scelte iniziali compiute in termini di tracciamento e di ospedalizzazione da parte di Regione Lombardia, fattori già richiamati da Quaglia, Terraneo e Tognetti. Tali scelte affondano le loro radici nel modello sanitario lombardo adottato nella seconda metà degli anni Novanta, che penalizza le cure primarie, l'assistenza extra-ospedaliera e il coordinamento con quella ospedaliera. Questa tesi, più volte citata in questi mesi, è supportata da Arlotti e Marzulli con l'elaborazione di dati quantitativi. La constatazione del fallimento della riforma del 2015 nell'intervenire su queste lacune pone degli interrogativi sulla capacità del processo di riforma attualmente in corso di rinnovare radicalmente le cure primarie e quelle di comunità. In questo senso, la sfida del sistema lombardo è prima di tutto culturale, in quanto deve essere in grado di recuperare la dimensione delle relazioni sociali, ossia «di qualcosa che viene prima del ricovero in ospedale e immediatamente dopo, all'atto della dimissione» (Arlotti, Marzulli, 2020). Solo così sarà possibile conseguire quel raccordo tra ospedale e territorio che sembra ancora mancare.

\section{Bibliografia}

Arlotti M., Marzulli M. (2020). La Regione Lombardia nella crisi sanitaria da Covid-19: ospedali, territorio e RSA. Milano: FrancoAngeli.

Baldi B., Profeti S. (2020). Le fatiche della collaborazione. Il rapporto stato-regioni in Italia ai tempi del COVID-19. Rivista Italiana di Politiche Pubbliche, 3: 277306. 
Biondi M., Iannitelli A. (2020). COVID-19 e stress da pandemia: l'integrità mentale non ha alcun rapporto con la statistica. Rivista di Psichiatria, 55(3): 31-136. DOI $10.1708 / 3382.33567$

Camilla A. (2020). Gli effetti psicologici della pandemia. Internazionale, 10 dicembre 2020.

Capano G. (2020). Policy design and state capacity in the COVID-19 emergency in Italy: if you are not prepared for the (un)expected, you can be only what you already are. Policy and Society, 39(3): 326-344. DOI: 10.1080/14494035.2020.1783790

Caporale C., Pirni A., a cura di (2020). Pandemia e resilienza. Persona, famiglia e comunità dopo la Covid 19. Roma: Cnr Edizioni.

Caritas (2020). Gli anticorpi della solidarietà. Rapporto 2020 su povertà ed esclusione sociale in Italia. Roma: Caritas Italiana.

Caruso C. (2021). Il regionalismo autarchico è incostituzionale: dal Giudice delle leggi una pronuncia che mette ordine nella gestione territoriale della pandemia. Questione Giustizia. Testo disponibile al sito: https://www.questionegiustizia.it/data/doc/2858/caruso_nota-qg.pdf (30/08/21).

Chicchi F., Simone A. (2017). La società della prestazione. Roma: Ediesse.

Conrad P. (2007). The Medicalization of Society: On the Transformation of Human Conditions into Treatable Disorders. Baltimore: The Johns Hopkins University Press.

Corposanto C., Fotino M., a cura di (2020). Covid 19. Le parole diagonali della Sociologia. Catanzaro: Thediagonales.

Corte dei Conti (2021). Rapporto di coordinamento sulla finanza pubblica. Roma: Corte dei Conti. Testo disponibile al sito: https://www.corteconti.it/Home/Documenti/RapportoCoordinamentoFP $(14 / 09 / 21)$.

Cozza M., Gherardi S., Graziano V., Johansson J., Mondon-Navazo M., Murgia A., Trogal K. (2020). COVID-19 as a breakdown in the texture of social practices. Gender, work and organization, 28(1): 190-208. DOI: 10.1111/gwao.12524

Favretto A., Maturo A., Tomelleri S. (2021). Introduzione. In: Favretto A., Maturo A., Tomelleri S., a cura di, L'impatto sociale del Covid-19. Milano: FrancoAngeli.

Hovanec C. (2011). Of Bodies, Families, and Communities: Refiguring the 1918 Influenza Pandemic. Literature and Medicine, 29(1): 161-181. DOI: $10.1353 / \mathrm{lm} .2011 .0314$

ISTAT (2020), Rapporto annuale 2020. La situazione del Paese. Roma.

ISTAT (2021). Le richieste di aiuto durante la pandemia. I dati dei centri antiviolenza, delle Case rifugio e delle chiamate al 1522. Anno 2020. Roma.

Kirchmayr R. (2020). Sulla miseria dell'attuale filosofia. Antinomie, 29 Aprile 2020.

Lupton D., Willis K. (2021). The Covid-19 crisis. Social perspectives. New York: Routledge. 
Manca A., Benczur P., Giovannini E. (2017). Building a Scientific Narrative Towards a More Resilient EU Society Part 1: A Conceptual Framework. Luxembourg: Publications Office of the European Union.

Mela A., Mugnano S., Olori D., a cura di (2017). Territori vulnerabili. Verso una nuova sociologia dei disastri italiana. Milano: FrancoAngeli.

Neri S. (2020). Più Stato e più Regioni. L'evoluzione della governance del Servizio sanitario nazionale e la pandemia. Autonomie Locali e Servizi Sociali, 2: 239-55.

Oecd (2021). Strengthening the frontline. How primary health care helps health systems adapt during the COVID 19 pandemic. Paris: Oced. Testo disponibile al sito: https:/www.oecd.org/coronavirus/policy-responses/strengthening-thefrontline-how-primary-health-care-helps-health-systems-adapt-during-thecovid-19-pandemic-9a5ae6da/ (30/08/2021)

Oxfam Italia (2021). Il virus della disuguaglianza. Un'economia equa, giusta e sostenibile per ricucire un mondo lacerato dal Coronavirus. Oxford: Oxfam International.

Pellizzoni L. (2020). La sfida del Covid-19 alle scienze umane. Alcune piste di riflessione. Le parole e le cose, 3 Aprile 2020.

Pfister S.M. (2020). Theorising - The Social Definition of the Corona Pandemic. The European Sociologist Issue 45: Pandemic (Im)Possibilities, 1. DOI: 10.1108/IJSSP-06-2020-0243

Rita V. (2021). Dopo il lockdown sono aumentati stress, ansia e sintomi depressivi. La Repubblica, 16 ottobre 2020.

Rosa H. (2010). Alienation and Acceleration: Towards a Critical Theory of LateModern Temporality. Malmö: Nordic Summer University Press.

Scambler G. (2020). Covid-19 as a "breaching experiment": exposing the fractured society. Health Sociology Review, 29(2): 140-148. DOI: 10.1080/14461242.2020.1784019

Sisto D. (2018). La morte si fa social. Immortalità, memoria e lutto nell'epoca della cultura digitale. Torino: Bollati Boringhieri.

Sità C. (2017). La genitorialità intensiva e le sue implicazioni per la relazione tra genitori e professionisti. Consultori Familiari Oggi, 25(2): 45-55.

Vicarelli G., Bronzini M. (2019). Introduzione. Sanità e innovazione tecnologica: un approccio pragmatico. In: Vicarelli G., Bronzini M., a cura di, Sanità digitale. Riflessioni teoriche ed esperienze applicative. Bologna: Mulino.

Vicarelli G., Neri S. (2021). Una catastrofe vitale? Le scelte di politica sanitaria per far fronte al Covid-19. Politiche sociali, 2: 233-254. 